\title{
Controlled motion of skyrmions in a magnetic antidot lattice
}

\author{
J. Feilhauer, ${ }^{1, *}$ S. Saha $\odot,{ }^{2,3, \dagger}$ J. Tobik $\odot,{ }^{1,4}$ M. Zelent $\odot,{ }^{5}$ L. J. Heyderman $\odot,,^{2,3}$ and M. Mruczkiewicz ${ }^{1,4, \sharp}$ \\ ${ }^{1}$ Institute of Electrical Engineering, Slovak Academy of Sciences, Dubravska Cesta 9, SK-841-04 Bratislava, Slovakia \\ ${ }^{2}$ Laboratory for Mesoscopic Systems, Department of Materials, ETH Zurich, 8093 Zurich, Switzerland \\ ${ }^{3}$ Laboratory for Multiscale Materials Experiments, Paul Scherrer Institute, 5232 Villigen PSI, Switzerland \\ ${ }^{4}$ Centre for Advanced Materials Application CEMEA, Slovak Academy of Sciences, Dubravska cesta 5807/9, 84511 Bratislava, Slovakia \\ ${ }^{5}$ Faculty of Physics, Adam Mickiewicz University in Poznan, Umultowska 85, Poznan, PL-61-614 Poland
}

(Received 18 October 2019; revised 4 September 2020; accepted 15 October 2020; published 19 November 2020)

\begin{abstract}
Future spintronic devices based on skyrmions will require precise control of the skyrmion motion. We show that this goal can be achieved through the use of magnetic antidot arrays. We perform micromagnetic simulations and semianalytical calculations based on the Thiele equation, where the skyrmion motion is driven by applied electric current via spin transfer torque (STT) or spin orbit torque (SOT) mechanism. For both torque mechanisms we demonstrate that an antidot array can guide the skyrmions in different directions depending on the parameters of the applied current pulse. Despite the fixed direction of the net driving current, due to the nontrivial interplay between the repulsive potential introduced by the antidots, the skyrmion Hall effect, and the nonuniform current distribution, full control of skyrmion motion in a 2D lattice can be achieved. Moreover, we demonstrate that the direction of skyrmion motion can be controlled by tuning only a single parameter of the current pulse, i.e., current magnitude.
\end{abstract}

DOI: 10.1103/PhysRevB.102.184425

\section{INTRODUCTION}

In the approximation of continuous magnetization, magnetic skyrmions [1-3] are topologically nontrivial stable spin textures that can be characterized by a nonzero winding number. The winding number for skyrmions in 2D films is $w=$ \pm 1 , which signifies that the magnetic state of the skyrmion cannot be unwound into a homogeneous state by a continuous transformation. Skyrmions in ferromagnetic materials are stabilized by the interplay of several magnetic energy contributions, i.e., exchange, dipole, anisotropy, and Zeeman. In particular, the asymmetric Dzyaloshinskii-Moriya exchange interaction (DMI) introduces chiral canting between neighboring spins and favors skyrmion stability [4,5]. DMI can arise due to the spin-orbit coupling and the lack of structural inversion symmetry, which can be realized in asymmetric heavy metal/ferromagnet heterostructures [6]. Due to the interfacial anisotropy, the ferromagnetic layer is magnetized in out-of-plane and the skyrmion is a defect in this homogeneous magnetization with diameter from a few to several hundreds of nanometers. The magnetization of the skyrmion center is oriented in the opposite direction with respect to the rest of the ferromagnetic layer and the magnetization of skyrmion continuously rotates from the center to its edges, so reversing in a radially symmetric fashion.

Another appealing feature of magnetic skyrmions is the possibility to manipulate them by electric current $[7,8]$ via

\footnotetext{
*juraj.feilhauer@savba.sk

${ }^{\dagger}$ susmitasaha.phy@gmail.com

‡michal.mruczkiewicz@savba.sk
}

spin transfer [9-11] and spin orbit torques [12-14]. In general, the current density required to drive the skyrmion motion is much smaller than the current density required to move a domain wall [15-17]. These unique features of skyrmions make them a promising candidates for future spintronic applications such as low dissipation magnetic information storage devices [18], skyrmion racetrack memories [19], and logic devices [20].

Unfortunately, skyrmions driven by the electric current migrate towards the edges of a magnetic strip, due to the skyrmion Hall effect, which leads to an unstable transverse position and annihilation. Furthermore, the skyrmion motion is randomized by the thermal diffusion and the presence of pining centers in real samples $[21,22]$. Therefore, key challenges regarding skyrmion motion are related to stabilization, confinement, and control of the movement of skyrmions at room temperature $[23,24]$. The observation that the edges of the sample and defects repel skyrmions led to the idea to use periodic lattices as a medium with well defined and robust motion of skyrmions [13,21].

In order to achieve the control over the skyrmion motion, we consider an antidot lattice realized as a thin ferromagnet/heavy metal bilayer (e.g., $\mathrm{Co} / \mathrm{Pt}, \mathrm{CoFeB} / \mathrm{Pt}$ ) with circular holes arranged on periodic square lattice. A hole in the magnetic texture of the ferromagnetic layer (i.e., region with zero magnetization) can be viewed as a homogeneous layer superposed with a disk with the opposite magnetization orientation. Since the magnetization of this disk is oriented in the same direction as the magnetization of the skyrmion center, the skyrmion is magnetostatically repelled by the antidot. The repulsion of skyrmion by the antidot lattice can therefore be expressed by a periodic effective potential with 
energy minima (valleys) located between each four neighboring antidots. Due to the dissipation, in the absence of driving torques acting on the skyrmion, the skyrmions are stabilized near the bottom of the valleys. In other words, in the presence of the antidot lattice, the relaxed positions of skyrmions are confined to the discrete square lattice of valleys as was already demonstrated both theoretically and experimentally in [25]. Sufficient spin torque is required to move the skyrmions over the energy barrier from one valley to another. In this paper, we separately study the effect of spin transfer torque (STT) generated by electric current flowing directly in the ferromagnetic layer and spin orbit torque (SOT) arising from an out-of-plane spin current generated via the spin Hall effect with an electric current flowing in the heavy metal layer. The STT or SOT acting on the skyrmions can be tuned with current pulses of different density and pulse width. Due to the discrete nature of the valleys, the current pulse parameters required for a skyrmion to arrive at a particular valley create compact regions in the parameter plane encompassing the pulse width $\Delta t$ and the current density $j$. Employing a micromagnetic solver Mumax3 [26] and a semianalytical model based on the Thiele equation [27], we calculate a map of these regions in the $(\Delta t, j)$ plane and study its changes upon variation of system properties such as damping.

The skyrmion trajectories in general are not parallel to the current density due to the skyrmion Hall effect. For the case of the skyrmion motion driven by STT, due to the nontrivial interplay between the skyrmion Hall effect and the potential landscape of the antidots, for shorter current pulses with larger magnitudes, the skyrmion can be moved to the valleys in the direction strictly parallel to the net applied current. Thus, for a particular range of current pulse parameters, the skyrmion Hall effect is effectively suppressed. By applying longer current pulses with smaller magnitudes, the skyrmion can be moved into specific valleys in the direction perpendicular to the current. For this range of current parameters the skyrmion Hall effect is enhanced.

For the case of the skyrmion motion driven by SOT, for shorter current pulses with larger magnitudes, the skyrmion can be moved to the valleys in the direction perpendicular to the net applied current, i.e., the skyrmion Hall effect is effectively enhanced. By applying a longer current pulses with smaller magnitudes, the skyrmion can be moved into specific valleys in the direction strictly parallel to the current, i.e., the skyrmion Hall effect is suppressed.

For both spin torque mechanisms, we demonstrate that, for an adequate combination of material, current and antidot lattice parameters, the skyrmion can be transported to almost any nearest-neighboring valley by applying a specific current pulse. Moreover, transport to the nearest-neighboring valleys can be achieved by tuning a single parameter of the current pulse, namely the current magnitude.

We therefore propose a method for unprecedented control of the skyrmion placement, utilizing the induced periodic potential of the antidot lattice. This is an important step towards exploiting antidot arrays in future skyrmion based spintronic devices.

The paper is organized as follows. In Sec. II, we introduce the numerical and semianalytical models for skyrmion transport in the antidot lattice driven by an applied current. We consider STT and SOT mechanisms separately. In Sec. III, we discuss the results in three parts. To identify some characteristic features of the skyrmion transport in the physical system, we first study a much simpler model with negligible damping and spatially uniform current density (Sec. III A). Then we introduce damping into the model and study the interplay between the potential of the antidot lattice and the skyrmion Hall effect (Sec. III B). Finally, we utilize the gained knowledge to describe the skyrmion transport in the physical system with damping and nonuniform current density resulting from the presence of the antidots (Sec. III C).

\section{METHODS}

To study the current driven dynamics of skyrmions in the antidot lattice, we have used the following two methods. First, we employed the Mumax 3 solver to perform micromagnetic simulations, which is a proven method for the investigation of magnetization processes. Second, the soliton nature of the skyrmion facilitates use of a simpler formalism than that used by the micromagnetic simulations to sufficiently describe the skyrmion dynamics, i.e., the Thiele equation. The Thiele equation is solved by numerical time integration. In this section we provide a detailed description of the calculations for both approaches.

\section{A. Micromagnetic simulations}

The governing equation of magnetization dynamics is the Landau-Lifshitz-Gilbert (LLG) equation, which is a partial differential equation numerically solved by Mumax 3 via a finite difference method [26]. To significantly reduce the duration of skyrmion transport simulations, we have used periodic boundary conditions in our micromagnetic calculations which is justified by the fact that the distribution of antidots as well as of electric current density is periodic within the antidot lattice. A unit cell of the antidot lattice has a square shape with a side length (i.e., lattice constant) of $a=512 \mathrm{~nm}$ and thickness $h=1.4 \mathrm{~nm}$. To evaluate a long-range magnetostatic field in a unit cell we have used a procedure standardly implemented in Mumax3, i.e., a finite number $N$ of unit cell images (copies) repeated in each planar direction is taken into account. We set $N=3$ which is, with respect to the size of our unit cell $a$, sufficient to obtain a periodiclike magnetostatic field. The unit cell contains one antidot realized as a circular hole with diameter $d=250 \mathrm{~nm}$. The rectangular regular discretization mesh has dimensions $2 \times 2 \times 1.4 \mathrm{~nm}^{3}$. The simulated material was $\mathrm{CoFeB}$ with material parameters: saturation magnetization $M_{S}=1.2 \times 10^{6} \mathrm{~A} / \mathrm{m}$, out-of-plane uniaxial anisotropy constant $K_{1}=9 \times 10^{5} \mathrm{~J} / \mathrm{m}^{3}$, DMI constant $D=1 \mathrm{~mJ} / \mathrm{m}^{2}$, exchange constant $A_{e x}=1.5 \times 10^{-11}$ $\mathrm{J} / \mathrm{m}$, and damping constant $\alpha$ in the interval from 0.03 to 0.3 . To stabilize the skyrmions an out-of-plane magnetic field of $30 \mathrm{mT}$ was applied.

Applying an in-plane electric current, the skyrmion motion can be induced by two main mechanisms as follows. For the case of the spin transfer torque (STT) the electric current flows directly in the ferromagnetic layer and its interaction with magnetization can be adequately modelled by a Zhang-Li form of the current-induced STT [28]. We neglect the nonadi- 


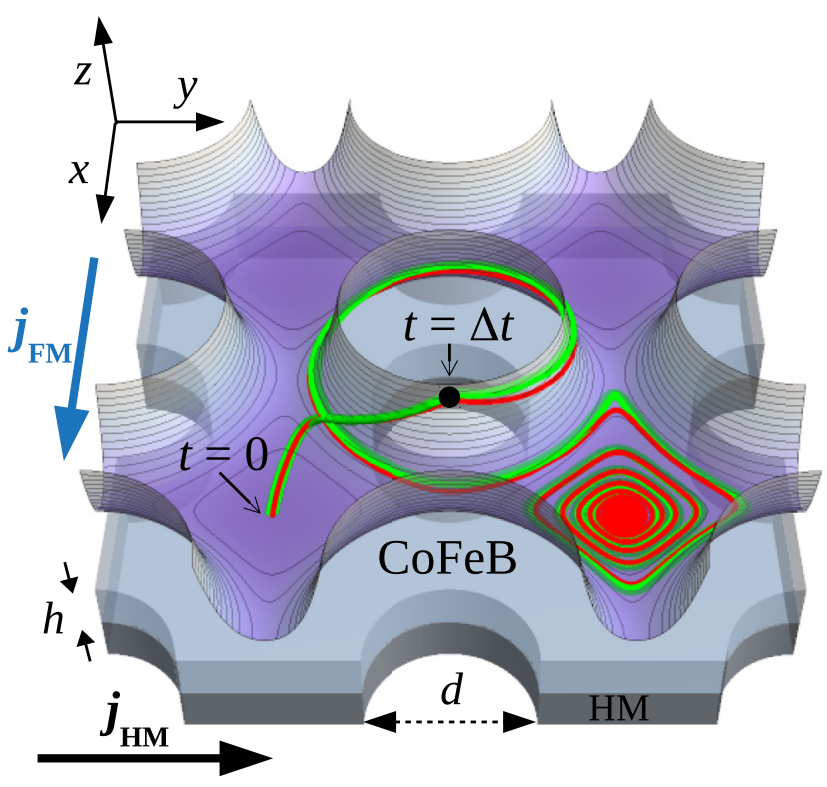

FIG. 1. Schematic of the ferromagnet/heavy metal heterostructure that hosts the magnetic skyrmions. Antidots are given by circular holes with diameter $d=250 \mathrm{~nm}$ arranged in the square lattice with lattice constant $a=512 \mathrm{~nm}$. The antidots repel the skyrmions as indicated by the potential $V(x, y)$ plotted above the heterostructure. The color curves are an example of a skyrmion trajectory induced by applying the current pulse $\mathbf{j}_{\mathrm{FM}}$ in the $x$ direction $\left(j_{\mathrm{FM}}=100 \mathrm{GA} / \mathrm{m}^{2}\right)$ or $\mathbf{j}_{\mathrm{HM}}$ in the $y$ direction $\left(\theta_{\mathrm{SH}} j_{\mathrm{HM}}=4.13 \mathrm{GA} / \mathrm{m}^{2}\right)$. The skyrmion is driven from the relaxed position at the potential minimum (at $t=0$ ) to the antidot wall. When the current pulse is switched off at $t=$ $\Delta t=62 \mathrm{~ns}$ (black point), the skyrmion orbits around the antidot and simultaneously relaxes to the neighboring potential minimum. The trajectory calculated by Thiele equation (red curve) is in a reasonable agreement with the trajectory calculated micromagnetically (green curve).

abatic contribution to the torque by setting the nonadiabaticity parameter to $\xi=0$ [26]. The current densities of rectangular current pulses used in the simulations were varied up to $400 \mathrm{GA} / \mathrm{m}^{2}$, which are the values considered in the experiments on skyrmion motion induced by charge current [29]. The net current $j_{\mathrm{FM}}$ was applied in the form of a rectangular pulse in the direction parallel to the row of antidots, along the $x$ axis (see Fig. 1).

For the case of the spin orbit torque (SOT), the electric current $j_{\mathrm{HM}}$ flows in the underlying heavy metal layer and the torque acting on the magnetization texture of the ferromagnet is generated by an out-of-plane spin current resulting from the spin Hall effect. In LLG equation, the SOT can be modeled by a Slonczewski-like term with the input parameters being the magnitude of the out-of-plane spin current $\theta_{\mathrm{SH}} j_{\mathrm{HM}}$, where $\theta_{\mathrm{SH}}$ is the spin Hall angle and a polarization of the spin current oriented in plane.

\section{B. Thiele equation}

The soliton character of the skyrmion means that it can be described as a quasiparticle with a fixed magnetization profile $\mathbf{m}_{s k}$ (with $\left|\mathbf{m}_{s k}\right|=1$ ). This approach considerably reduces the multidimensional spin degrees of freedom, leaving only two parameters-the coordinates of skyrmion center $\mathbf{r}=(x, y)$. The quasiparticle approximation of the skyrmion transforms the LLG equation into the Thiele equation [27], which is an ordinary differential equation describing the dynamics of the skyrmion center located at $\mathbf{r}$. The translational motion of a skyrmion is affected by the interaction with antidots, which can be represented by a repulsive potential $V(\mathbf{r})$. The motion is driven by the current density $\mathbf{j}(\mathbf{r}, t)$ which, for the case of a rectangular current pulse with width $\Delta t$, is given by $\mathbf{j}(\mathbf{r}, t)=\mathbf{j}(\mathbf{r}) \Theta(\Delta t-t)$, where $\Theta$ is the Heaviside step function. All these effects are included in the modified Thiele equation $[10,27,30]$, which is usually written in the form

$$
\mathbf{G} \times \dot{\mathbf{r}}-\alpha \mathbf{D} \dot{\mathbf{r}}+\mathbf{F}_{\mathbf{j}}+\mathbf{F}_{a d}=0,
$$

where $\mathbf{G}$ is the gyromagnetic coupling vector, $\alpha$ is the magnetic damping, and $\mathbf{D}$ is the dissipative force tensor.

The first term in (1) is the topological Magnus force, perpendicular to the velocity of the skyrmion, which is responsible for the gyrotropic (transverse) motion of the skyrmion $[12,31]$. In ultrathin films, the magnetization is constant in the $z$ direction and the gyromagnetic coupling vector $\mathbf{G}$ is perpendicular to the film plane, i.e., $\mathbf{G}=G \mathbf{e}_{z}$ with

$$
G=-\iint \mathbf{m}_{s k}\left[\frac{\partial \mathbf{m}_{s k}}{\partial x} \times \frac{\partial \mathbf{m}_{s k}}{\partial y}\right] d x d y=-4 \pi w,
$$

where the winding number $w$ also represents the topological charge of the skyrmion independent of the skyrmion dimensions. We assume that the magnetization of the skyrmion center is oriented in the negative $z$ direction. Therefore the winding number of skyrmion used in Eq. (2) is $w=-1$.

The second term in (1) is the dissipative force, which is linked to the damping in the material and responsible for the friction acting on the skyrmion. In an ultrathin film, the dissipative force tensor $\mathbf{D}$ is diagonal and has the form $\mathbf{D}=D \delta_{i j}$ with

$$
D=\iint \frac{\partial \mathbf{m}_{s k}}{\partial x} \cdot \frac{\partial \mathbf{m}_{s k}}{\partial x} d x d y .
$$

Typically, $D$ is equal to several units of $\pi$ (see, e.g., Ref. [32]) and we use $D=5.73 \pi$, which provides the best fit of our Thiele equation results with the data obtained by micromagnetic simulations.

Modeling the skyrmion magnetization profile as a $360^{\circ}$ Néel domain wall, i.e., $\mathbf{m}_{s k}=(\cos \varphi \sin \theta, \sin \varphi \sin \theta, \cos \theta)$ with $\tan \varphi=y / x$ and

$$
\theta(r)=2 \arctan \left[\frac{\sinh \left(R_{s k} / \Delta\right)}{\sinh (r / \Delta)}\right],
$$

where $r=\sqrt{x^{2}+y^{2}}, R_{s k}$ is a radius of the skyrmion and $\Delta$ is the width of the domain wall, we can rewrite the integral in (3) into the form

$$
D=\pi \int_{0}^{\infty}\left[\left(\frac{\partial \theta}{\partial r}\right)^{2}+\frac{\sin ^{2} \theta}{r^{2}}\right] r d r .
$$

For $R_{s k} \gg \Delta$ the integral (5) can be analytically estimated [33] by the formula

$$
D \approx 2 \pi\left(\frac{R_{s k}}{\Delta}+\frac{\Delta}{R_{s k}}\right) .
$$


Employing the formula (4), we have fitted the micromagnetically calculated magnetization profile of a skyrmion located close to the center between the antidots. The resulting values of skyrmion radius and domain wall width were found to be $R_{s k}=43.2 \mathrm{~nm}$ and $\Delta=17 \mathrm{~nm}$. Inserting these values into the formula (6) yields $D=5.88 \pi$ which is in good agreement with the value used in our semianalytical calculations.

The third term in (1) is related to the force acting on the skyrmion as a result of the applied in-plane electric current. When considering the current flowing directly in the ferromagnetic layer $\left(\mathbf{j}_{\mathrm{FM}}\right)$, the force is generated via spin-transfer torque (STT) mechanism and has the form

$$
\mathbf{F}_{\mathbf{j}}{ }^{\mathrm{STT}}=\mathbf{G} \times b_{j} \mathbf{j}_{\mathrm{FM}}(\mathbf{r}, t),
$$

where $b_{j}=\left|\mu_{B} / e M_{S}\right|, \mu_{B}$ is the Bohr magneton, and $e$ is the elementary charge. However, taking into account the spin Hall effect occurring when the electric current flows through the heavy metal layer $\left(\mathbf{j}_{\mathrm{HM}}\right)$, the out-of-plane spin current is generated which creates a spin orbit torque (SOT) on the skyrmion generating a force $[31,34]$

$$
\mathbf{F}_{\mathbf{j}}{ }^{\text {SOT }}=\frac{\gamma \hbar C}{2|e| M_{S} h} \theta_{\mathrm{SH}} \mathbf{j}_{\mathrm{HM}}(\mathbf{r}, t),
$$

where $\gamma=g\left|\mu_{B}\right| / \hbar$ is a gyromagnetic ratio, $g$ is an electron $g$ factor, $\theta_{\mathrm{SH}}$ is a spin Hall angle of the heavy metal substrate, and

$$
C=\iint\left(m_{z}^{s k} \frac{\partial m_{x}^{s k}}{\partial x}-m_{x}^{s k} \frac{\partial m_{z}^{s k}}{\partial x}\right) d x d y .
$$

Employing the model (4), the integral (9) can be rewritten in the form

$$
C=\pi \int_{0}^{\infty}\left[\frac{\partial \theta}{\partial r}+\frac{\sin 2 \theta}{2 r}\right] r d r
$$

For $R_{s k} \gg \Delta$, the formula (10) can be simplified [33] to $C \approx$ $\pi^{2} R_{s k}$.

To simplify the discussion we treat both of the driving mechanisms separately. We note that according to (7), for the case of STT, the force $\mathbf{F}_{\mathbf{j}}$ STT acting on the skyrmion is perpendicular to the electric current $\mathbf{j}_{\mathrm{FM}}$, while for the case of the SOT [Eq. (8), the force $\mathbf{F}_{\mathbf{j}}{ }^{\text {SOT }}$ and electric current $\mathbf{j}_{\mathrm{HM}}$ are parallel.

For the case of a spatially uniform current, it is possible to obtain an identical skyrmion transport with STT and SOT driving mechanisms. Namely, we assume a constant force $\mathbf{F}_{\mathbf{j}}$ applied in the $y$ direction that, in the case of STT, corresponds to the electric current $\mathbf{j}_{\mathrm{FM}}$ flowing in the ferromagnetic layer along the $x$ direction and, in the case of SOT, to the current $\mathbf{j}_{\mathrm{HM}}$ flowing in the heavy metal layer along the $y$ direction. In other words, for both driving mechanisms, we can obtain identical skyrmion trajectories by adjusting the current magnitudes via $j_{\mathrm{FM}}=(g C / 8 \pi h) \theta_{\mathrm{SH}} j_{\mathrm{HM}}$, which leads to $\mathbf{F}_{\mathbf{j}}=\mathbf{F}_{\mathbf{j}}{ }^{\mathrm{STT}}=\mathbf{F}_{\mathbf{j}}{ }^{\mathrm{SOT}}$.

The fourth term in (1) represents the force acting on the skyrmion due to the repulsive interaction with antidot lattice, i.e.,

$$
\mathbf{F}_{a d}=-\frac{\gamma}{M_{s} h} \nabla V(\mathbf{r})
$$

The corresponding periodic potential $V(\mathbf{r})$ was constructed numerically with the use of the micromagnetic solver in the following manner: (i) a continuous current was applied to move the skyrmion out of equilibrium to higher energy and the magnetic state was recorded. (ii) The saved states were used as the initial states for the time evolution with reduced damping constant $\alpha=0.001$ in the absence of current. Each simulation generated quasi-isoenergy contours. (iii) The generated energy as a function of position was interpolated using a smooth function $V(x, y)$ which has the same periodicity as the corresponding antidot lattice.

The resulting periodic repulsive potential $V(x, y)$ is plotted in Fig. 1 with the minimas (valleys) centered between four neighboring antidots and saddles located between neighboring antidots. After algebraic manipulation, Eq. (1) can be written in the form

$$
\dot{\mathbf{r}}(t)=\mathcal{A} \mathbf{F},
$$

where the net force acting on the skyrmion reads $\mathbf{F}=\mathbf{F}_{\mathbf{j}}+$ $\mathbf{F}_{a d}$ and

$$
\mathcal{A}=\frac{1}{G^{2}+\alpha^{2} D^{2}}\left[\begin{array}{cc}
\alpha D & -G \\
G & \alpha D
\end{array}\right]
$$

We solved the set of Eq. (12) numerically with the initial condition $\mathbf{r}(t=0)$ set at the lowest point of the potential landscape $V(\mathbf{r})$, which corresponds to the relaxed position of the skyrmion without an external driving force.

The applicability of the Thiele equation approach was tested by comparing the skyrmion trajectories driven by STT and SOT with calculations performed using the micromagnetic solver for various values of current density and the pulse width, and we found that the trajectories are in agreement. Examples of the two trajectories determined with the different methods are shown in Fig. 1.

With the use of the Thiele equation, we determined the final position of the skyrmion after the application of a current pulse and constructed a map of the final skyrmion positions as the function of the current density and the pulse width. Applying strong current density might lead to skyrmion annihilation at the antidot boundaries. However, the process of annihilation of the skyrmion by pushing it to an antidot is beyond the validity of the Thiele equation approach. Therefore, we have used micromagnetic simulations to determine the critical values of the current pulse parameters, where the annihilation starts to occur.

The antidots realized as holes in the layers do not conduct electric current. The spatially nonuniform current density distribution for a geometry with holes was calculated using Comsol. The nonuniform current density was implemented in the micromagnetic as well as in the Thiele equation calculations. However, the approximation of the uniform current density simplifies the interpretation of results and is valuable for a qualitative understanding of the magnetic processes. Therefore, in the following we present results for both uniform and nonuniform current distributions.

\section{RESULTS}

To understand the origin of several characteristic features of the skyrmion transport in a real system with damping and nonuniform current distribution, we first study a much simpler model with negligible damping and uniform current density 
(a)
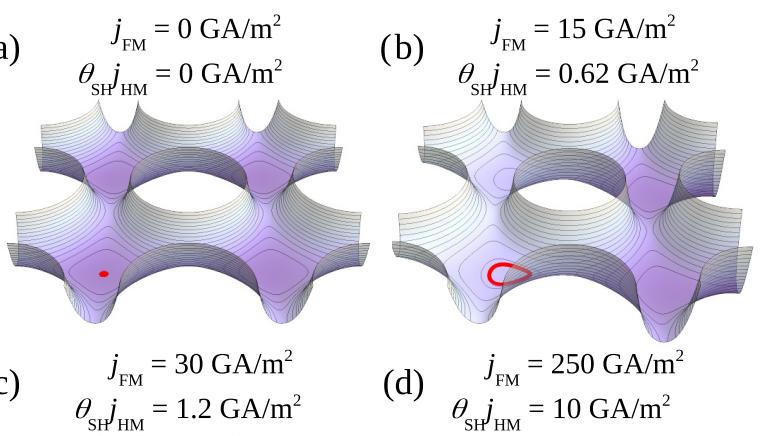

(c)

$$
\begin{aligned}
j_{\mathrm{FM}} & =30 \mathrm{GA} / \mathrm{m}^{2} \\
\theta_{\mathrm{SH}} j_{\mathrm{HM}} & =1.2 \mathrm{GA} / \mathrm{m}^{2}
\end{aligned}
$$
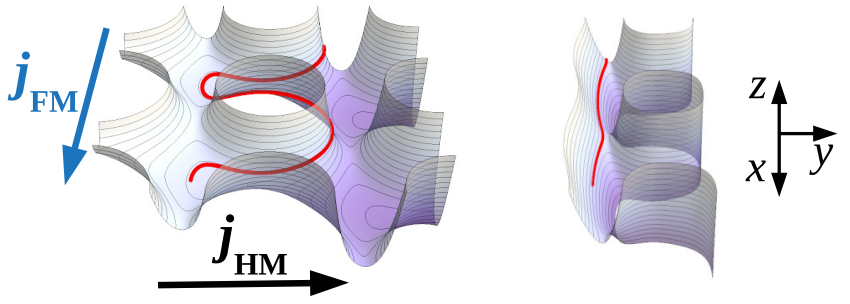

FIG. 2. Effective potential $V_{\text {eff }}$ combining the effects of antidot potential $V$ and driving current on the skyrmion motion in the undamped sample. (a)-(d) With increasing $\mathbf{j}_{\mathrm{FM}}$ or $\mathbf{j}_{\mathrm{HM}}$, the tilt of $V_{\mathrm{eff}}$ along the $y$ direction increases. Red curves show the corresponding skyrmion trajectories starting from the potential minimum (valley) of $V$.

(Sec. III A). Then we introduce damping into the model and study its effect on the skyrmion trajectory and final position after the current pulse is applied (Sec. III B). Finally, to determine the behavior of skyrmions in a physical system, we incorporate a nonuniform current distribution into the model and discuss the similarities and differences with the previous simpler cases (Sec. III C).

\section{A. Damping free system with uniform current}

The main goal of this paper is to study the transport of skyrmions driven by the applied current pulse in the presence of a repulsive antidot lattice. For simplicity, we start with the damping free material, i.e., we set $\alpha=0$. In this case, the diagonal elements of the matrix $\mathcal{A}$ in (13) are zero, which means that we can rewrite the equation of motion (12) as

$$
\dot{\mathbf{r}}(t)=\left(\mathbf{e}_{z} \times \mathbf{F}\right) / G .
$$

Moreover, if we assume that the current density is spatially uniform, the effect of current can be easily incorporated into the potential via an extra term $V_{j}=-\frac{M_{s} h}{\gamma} \mathbf{r} . \mathbf{F}_{\mathbf{j}}$ yielding $\mathbf{F}=-\frac{\gamma}{M_{s} h} \nabla V_{\text {eff }}$, where $V_{\text {eff }}=V+V_{j}$. The dynamics of the skyrmion described by Eq. (14) can be readily understood, since the skyrmion moves perpendicularly to the force $\mathbf{F}$, i.e., it simply follows the isoenergy contour of the effective potential $V_{\text {eff. }}$.

When the current is switched off, $V_{\text {eff }}=V$, with closed isoenergy contours centered around the bottom of the valley or around an antidot, depending on whether the corresponding energy is smaller or larger than the energy of the saddle point between the valleys [see Fig. 2(a). When the uniform current is switched on, the resulting effective potential $V_{\text {eff }}$ is just the antidot potential $V$ tilted in the direction of the current-induced force $\mathbf{F}_{\mathbf{j}}$. The amount of tilt is proportional to the magnitude of the current density, i.e., the larger the current, the larger the tilt of the antidot potential. In particular, as discussed in the previous section, when the current flows along the $x$ direction for the case of STT [i.e., $\mathbf{j}_{\mathrm{FM}}=\left(j_{\mathrm{FM}}, 0\right)$ ] or along the $y$ direction for the case of SOT [i.e., $\mathbf{j}_{\mathrm{HM}}=$ $\left.\left(0, j_{\mathrm{HM}}\right)\right]$, the current-induced force is oriented along the $y$ direction, i.e., $\mathbf{F}_{\mathbf{j}}=\left(0, F_{j}\right)$. Then the effective potential can be simplified to

$$
V_{\text {eff }}(x, y)=V(x, y)-\frac{M_{s} h}{\gamma} y F_{j},
$$

i.e., it is equal to the antidot potential $V$ tilted in the $y$ direction. The isoenergy contours of this tilted $V_{\text {eff }}$ are no longer just closed but open runaway contours also exist and extend between the valleys in the $x$ direction [see Figs. 2(b)-2(d).

We now discuss the three types of skyrmion trajectories resulting from the applied current, assuming the skyrmion starts from the bottom of the valley, i.e., the center between four neighboring antidots [red point in Fig. 2(a). For smaller current density, the corresponding isoenergy contour of $V_{\text {eff }}$ is closed and skyrmion oscillates inside the valley [Fig. 2(b). As the current density is increased, the tilt of the antidot potential can be large enough to cause the contour of $V_{\text {eff }}$ crossing the starting point to open. In this case, the skyrmion escapes the starting valley and passes along an antidot to the neighboring valley in the $x$ direction [Fig. 2(c). For sufficiently large current density, the tilt of the antidot potential is so large that the isoenergy contour along which the skyrmion passes to the neighboring valley can pass directly through the saddle between the valleys avoiding the passage of the skyrmion around the antidot [see Fig. 2(d).

Our aim is to determine, and therefore control, the final positions of the skyrmion after applying a rectangular current pulse with the width $\Delta t$. As shown above, when the current is switched on, the skyrmion follows the isoenergy contour of the tilted antidot potential $V_{\text {eff }}$. Subsequently, when the current pulse is switched off, the tilted antidot potential returns back to the original antidot potential (without current) and the skyrmion follows a closed isoenergy contour, i.e., it stays trapped inside a valley or orbits around an antidot depending on the parameters $j_{\mathrm{FM}}$ (or $j_{\mathrm{HM}}$ ) and $\Delta t$. Five examples of such trajectories are shown in Figs. 3(a)-3(e) for a skyrmion starting at the valley at $(x, y)=(0,0)$ driven by a current pulse with fixed width $\Delta t=100 \mathrm{~ns}$ and various current densities.

The final positions of skyrmion resulting from the applied current pulse are summarized in a pulse width-current density map in Fig. 3(f). This map was calculated using the Thiele equation (12). The regions of parameters $j_{\mathrm{FM}}$ (or $j_{\mathrm{HM}}$ ) and $\Delta t$ which correspond to the same final position of the skyrmion are given by the same color. The gray regions correspond to the case where the skyrmion orbits around the antidots marked I, II, or III [see, e.g., Fig. 3(b). The other colored regions correspond to the situation where the skyrmion ends up trapped inside a valley in the horizontal direction [e.g., at $(x, y)=$ $(0,0),(-1,0), \ldots]$. For simplicity, we plot only the data for the valleys with distance smaller than $3 a$ from the origin. The data for valleys further than $3 a$ are all located in the white region in Fig. 3(b). When the current density is small or the pulse is very short, the skyrmion stays pinned inside the starting valley 

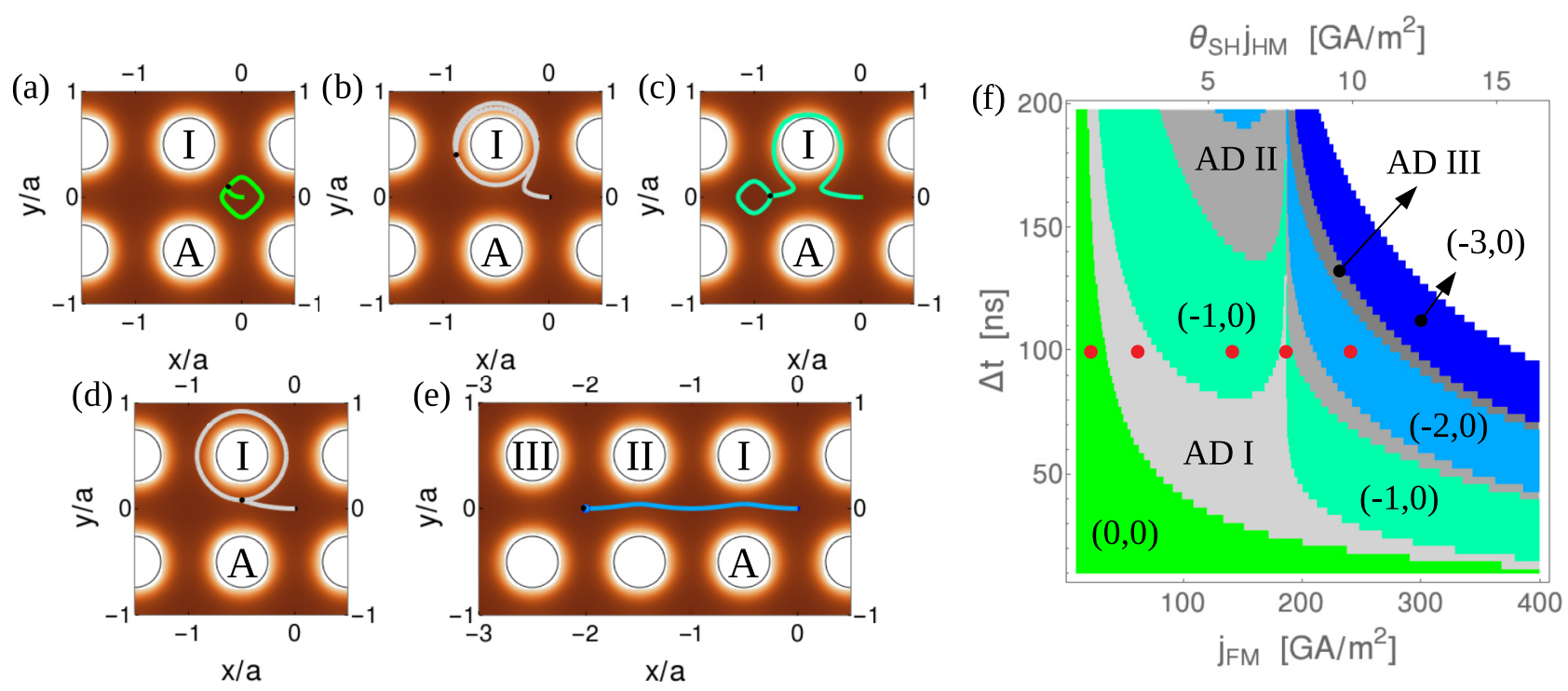

FIG. 3. Skyrmion transport without damping driven using rectangular current pulses with the width $\Delta t$. (a)-(e) Trajectories of skyrmions in the antidot array starting at the bottom of the valley at point $(x, y)=(0,0)$. The value of the pulse width is fixed to $\Delta t=100 \mathrm{~ns}$ and the values of $j_{\mathrm{FM}}$ are $20,60,140,187$, and $240 \mathrm{GA} / \mathrm{m}^{2}$ ( or $\theta_{\mathrm{SH}} j_{\mathrm{HM}}=0.82,2.5,5.8,7.7$, and $9.9 \mathrm{GA} / \mathrm{m}^{2}$ ), respectively. The color of each trajectory corresponds to a particular region in (f). Black dots denote the position of the skyrmion where the current pulse is switched off. (f) Map of the final positions of the skyrmion as a function of the current pulse width and density. Red dots indicate the parameters defining the trajectories in (a)-(e).

at $(0,0)$ as depicted by the dark green region. When increasing the pulse parameters, the current pulse is sufficient to move the skyrmion over the saddle point and the skyrmion orbits around the antidot I after the current pulse is turned off (light gray region, AD I). Increasing the current pulse parameters even more, the skyrmion is able to move to the neighboring valley [region $(-1,0)$ in light green], orbits around the antidot II (region AD II in dark gray), moves to the next-neigboring valley [region $(-2,0)$ in light blue] and so on. There is a discontinuity in Fig. 3(f) at $j_{\mathrm{FM}}^{u}=187 \mathrm{GA} / \mathrm{m}^{2}\left(\right.$ or $\theta_{\mathrm{SH}} j_{\mathrm{HM}}^{u}=$ $7.7 \mathrm{GA} / \mathrm{m}^{2}$ ), which separates two types of trajectories. For $j_{\mathrm{FM}}<j_{\mathrm{FM}}^{u}\left(\right.$ or $\left.j_{\mathrm{HM}}<j_{\mathrm{HM}}^{u}\right)$ the skyrmion trajectories crossing the valleys pass around the antidots I, II,... [e.g., the trajectory in Fig. 3(c)] while for $j_{\mathrm{FM}}>j_{\mathrm{FM}}^{u}$ (or $j_{\mathrm{HM}}>j_{\mathrm{HM}}^{u}$ ) the skyrmions reach these valleys directly through the saddles of the antidot potential [e.g., the trajectory in Fig. 3(e)]. At $j_{\mathrm{FM}}=$ $j_{\mathrm{FM}}^{u}\left(\right.$ or $\left.j_{\mathrm{HM}}=j_{\mathrm{HM}}^{u}\right)$ the skyrmion passes to the saddle point of the effective potential $V_{\text {eff }}$ located between the antidots I and A where $\mathbf{F}=\nabla V_{\text {eff }}=0$. Here, the torque acting on the skyrmion due to the applied current is completely compensated by the repulsion of the antidot lattice. Then, since the right side of the Thiele equation (14) vanishes, the skyrmion velocity becomes zero and it stops, i.e., the saddle point of $V_{\text {eff }}$ is an unstable position for the skyrmion. After the current pulse is switched off the skyrmion starts to orbit around the antidot I. The corresponding skyrmion trajectory is shown in Fig. 3(d) where the black point indicates the unstable position of the skyrmion where the skyrmion is fixed until the current pulse is switched off.

As shown above, without damping, for the case of the STT (or SOT) it would be possible to transfer the skyrmions only to neighboring valleys in the direction parallel (or perpendicular) to the current. As we show in the following section, to move the skyrmion to the valleys located at various angles with respect to the direction of the current, nonzero damping and the resulting skyrmion Hall effect is essential.

\section{B. Damped system with uniform current}

In order to determine how the motion of the skyrmion is affected by damping, we include a nonzero $\alpha$ in the off-diagonal elements of matrix $\mathcal{A}$ in Eq. (12). Then, in the unpatterned thin film, the skyrmion would move at an angle with respect to the current direction, which is the phenomenon known as the skyrmion Hall effect. For the case of STT, where the current flows along the $x$ direction, the skyrmion Hall angle is given by

$$
\left|\Theta_{H}^{\mathrm{STT}}\right|=\arctan \left|\frac{v_{y}}{v_{x}}\right|=\arctan \left|\frac{\alpha D}{G}\right|,
$$

i.e., at the negligible damping $\alpha$ (discussed in the previous subsection) the Hall angle is zero. From Eq. (16), we found that the value of $\Theta_{H}^{\mathrm{STT}}$ varies from $2.5^{\circ}$ to $23.3^{\circ}$ when changing $\alpha$ from 0.03 to 0.3 . For the case of SOT, where the current flows along the $y$ direction, the skyrmion Hall angle is given by

$$
\left|\Theta_{H}^{\mathrm{SOT}}\right|=\arctan \left|\frac{v_{x}}{v_{y}}\right|=\arctan \left|\frac{G}{\alpha D}\right|,
$$

i.e., at negligible damping $\alpha$, the Hall angle equals to $\pi / 2$. For $\alpha$ in the range $0.03-0.3$, the angle $\Theta_{H}^{\mathrm{SOT}}$ varies from $87.5^{\circ}$ to $66.7^{\circ}$.

Another effect resulting from the damping is the relaxation of the skyrmion to a lower energy configuration when the current pulse is switched off. Due to the damping, the skyrmion 


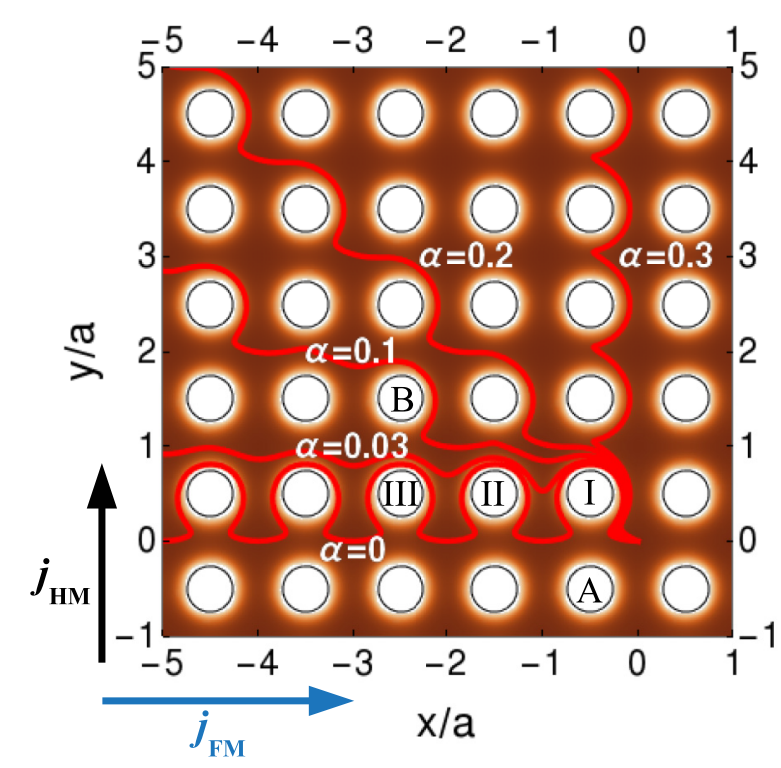

FIG. 4. Trajectories of a skyrmion starting in the valley at $(0,0)$ for various values of damping. The skyrmion motion is driven by the uniform current density $j_{\mathrm{FM}}=90 \mathrm{GA} / \mathrm{m}^{2}$ (or $\theta_{\mathrm{SH}} j_{\mathrm{HM}}=$ $3.7 \mathrm{GA} / \mathrm{m}^{2}$ ) applied along the $x$ (or $y$ ) direction. In the undamped case $(\alpha=0)$ the trajectory extends only to the valleys along the $x$ axis [e.g., at $(-1,0),(-2,0), \ldots$ ] while, for nonzero damping, the trajectories also reach the valleys in the various directions [e.g., at $(-1,1),(0,1), \ldots]$.

motion converges to the local energy minima, i.e., it ends up at the bottom of a valley.

Due to the nontrivial manifestation of damping in the Thiele equation (12), the simple concept of a tilted antidot potential $V_{\text {eff }}$ described in the previous subsection is not valid for nonzero damping. Therefore the shapes of the skyrmion trajectories are more complex, which is illustrated in Fig. 4 for fixed uniform current density $j_{\mathrm{FM}}=90 \mathrm{GA} / \mathrm{m}^{2}\left(\theta_{\mathrm{SH}} j_{\mathrm{HM}}=\right.$ $3.7 \mathrm{GA} / \mathrm{m}^{2}$ ) and various values of damping $\alpha$. In the undamped case $(\alpha=0)$, the trajectory extends in the $x$ direction with a periodic form with a period of a single lattice constant $a$. This is a result of the fact that the direction of the applied current and the corresponding force $\mathbf{F}_{\mathbf{j}}$ acting on the skyrmion are parallel to the symmetry axes of the antidot lattice. At finite damping, the skyrmion trajectory is tilted with respect to the $x$ direction as a result of the skyrmion Hall effect. Therefore, as was also shown in Ref. [13], depending on the amount of damping and density of the applied current, the skyrmion trajectories in the presence of an antidot lattice form a series of periodic discrete steps extending in both $x$ and $y$ directions with periods that can be multiples of $a$, e.g., the trajectory for $\alpha=0.1$ in Fig. 4.

For the case of the STT, with the increase of the damping, the angle between the skyrmion trajectory and the current direction along the $x$ axis (blue arrow in Fig. 4) also increases. Due to the presence of a periodic antidot potential, the trajectory angle is much larger than the corresponding Hall angle $\Theta_{H}^{\mathrm{STT}}$ for the unpatterned film. For example, the trajectory for $\alpha=0.3$ is perpendicular to the direction of the current while the corresponding Hall angle is only $23.3^{\circ}$. Therefore, for the case of STT the presence of the antidot lattice enhances the skyrmion Hall effect. Similar enhancement of skyrmion Hall effect was observed in Ref. [13] for a skyrmion moving on a two-dimensional periodic substrate.

For the case of the SOT, the skyrmion Hall effect is reduced in the presence of the antidot lattice, i.e., with increasing damping, the tilt of the trajectory with respect to the current $\mathbf{j}_{\text {HM }}$ (black arrow in Fig. 4) flowing along the $y$ direction decreases. For example, for $\alpha=0.3$ the skyrmion trajectory is almost perfectly aligned with the direction of the current.

For potential spintronic applications the skyrmion velocity is also an important factor. Therefore, to illustrate the effect of antidot lattice on skyrmion velocities, we compare the average velocities of skyrmions moving along the different trajectories in Fig. 4 with the velocities in a corresponding system without antidots calculated using the Thiele equation (14) for $V=0$. In the free system without antidots, the skyrmion velocities corresponding to the damping values $\alpha=$ $0,0.03,0.1,0.2,0.3$ are found to be $4.35,4.34,4.30,4.18$, $4.00 \mathrm{~m} / \mathrm{s}$, respectively. In the presence of the antidot lattice average skyrmion velocities increase by a factor of 2.17 , $1.94,1.49,1.53,1.29$, respectively, i.e., for zero damping the skyrmion velocity in the antidot lattice is increased roughly by a factor of two when compared with the free layer and with increasing damping this increase is reduced.

As we have seen, the skyrmion trajectories in a damped system extend not only to the valleys in the $x$ direction but also to the valleys at various angles with respect to the current direction. Therefore, the number of valleys that are reachable by the skyrmion is significantly increased. When considering a finite current pulse, we can distinguish two scenarios for the skyrmion transfer between the neighboring valleys. First, the skyrmion is directly transferred to the desired valley by an applied current and then, when the current is switched off, the skyrmion relaxes to the valley bottom. The second case is illustrated in Fig. 1 where, after the current pulse is switched on, the skyrmion climbs from the bottom of the valley to the antidot wall until the pulse is switched off (black point). Without damping the skyrmion would orbit around the antidot forever but, since damping is present $(\alpha=0.03)$, the skyrmion looses its energy and relaxes in a spiral trajectory to the bottom of one of the four valleys next to the antidot.

As for the undamped case in Fig. 3(b), in Fig. 5 we show the final positions of the skyrmion that result after applying a uniform current pulse with density $j_{\mathrm{FM}}$ (or $j_{\mathrm{HM}}$ ) and width $\Delta t$. The maps in Fig. 5 were calculated using the Thiele equation (12) for various values of damping constant $\alpha$. For small damping, $\alpha=0.03$ [Fig. 5(a), we can identify several features that also appear in the undamped case. When the current density or width of the current pulse is too small, the skyrmion just stays pinned in the starting valley (dark green region) after the pulse is applied. The current $j_{\mathrm{FM}}^{u}\left(\right.$ or $j_{\mathrm{HM}}^{u}$ ) defining the trajectories passing to the unstable position of the skyrmion located between the antidots I and $\mathrm{A}$ is shifted to the larger values, i.e., $j_{\mathrm{FM}}^{u}=234 \mathrm{GA} / \mathrm{m}^{2}$ (or $\theta_{\mathrm{SH}} j_{\mathrm{HM}}^{u}=9.6 \mathrm{GA} / \mathrm{m}^{2}$ ). For $j_{\mathrm{FM}}>j_{\mathrm{FM}}^{u}$ (or $j_{\mathrm{HM}}>j_{\mathrm{HM}}^{u}$ ), the region corresponding to the transport of skyrmion to the neighboring valley $(-1,0)$ directly through the saddle between the antidots I and A has almost the same area and shape as in the undamped case. However, due to the modified skyrmion Hall effect, the same valley is almost unreachable via the trajectories passing 

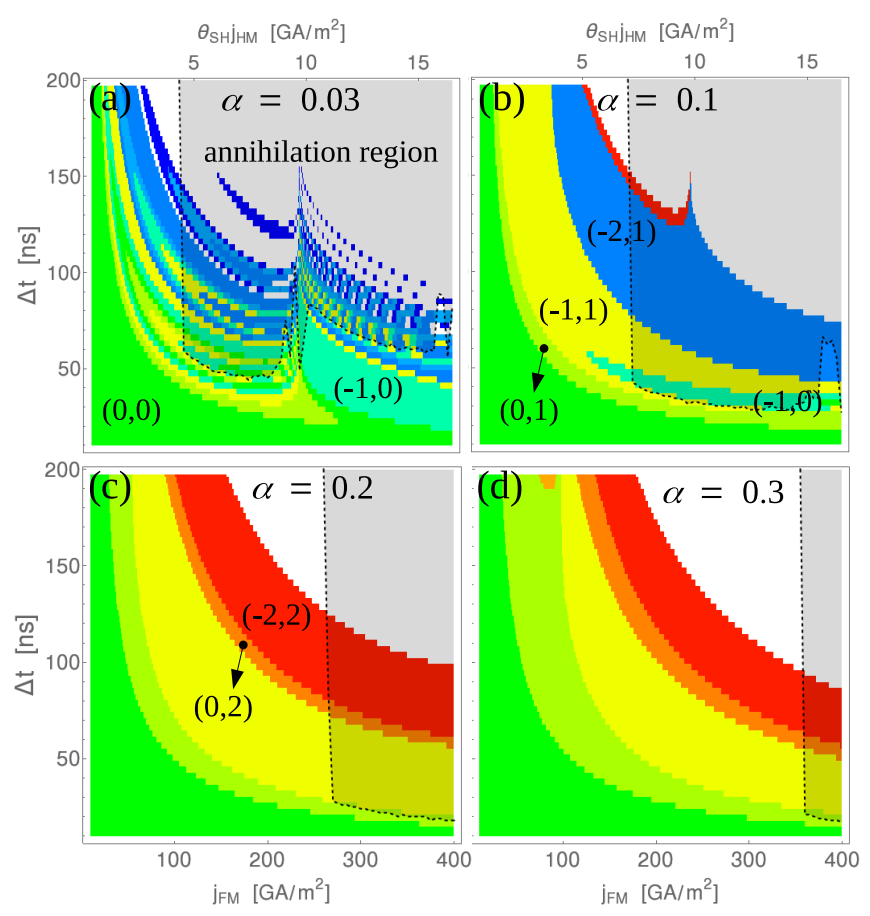

FIG. 5. Final position of the skyrmion after the application of uniform current pulse with density $j_{\mathrm{FM}}$ (or $j_{\mathrm{HM}}$ ) and width $\Delta t$. Color regions correspond to the parameters with the same final valley. The damping is (a) $\alpha=0.03$, (b) $\alpha=0.1$, (c) $\alpha=0.2$, and (d) $\alpha=0.3$. Shaded region calculated by micromagnetic solver shows the parameters of current pulse at which the skyrmion is annihilated.

around the antidot $\mathrm{I}$ and the region $(-1,0)$ is much smaller for $j_{\mathrm{FM}}<j_{\mathrm{FM}}^{u}$ (or $j_{\mathrm{HM}}<j_{\mathrm{HM}}^{u}$ ) than for the undamped case. The former light gray region ascribed to the trajectories orbiting around the antidot I [region AD I in Fig. 3(f) is now decomposed into the set of thin regions corresponding to the four valleys at $(x, y)=(0,0),(-1,0),(-1,1)$, and $(0,1)$ next to the antidot I. As discussed above, due to the damping, the skyrmion orbiting around the antidot I looses its energy and ends up in one of the four neighboring valleys. When the damping is small, the skyrmion can encircle the antidot several times until it relaxes to the final valley. Therefore, the final valley is strongly sensitive to the position of the skyrmion on the antidot wall at the moment when the current pulse is switched off. Similarly, the other gray regions from Fig. 3(c) describing the orbiting skyrmion around the antidots II, III,... decompose into the regions corresponding to the surrounding valleys. This is the reason for rather chaotic structure of the map in Fig. 5(a) for larger values of parameters $j_{\mathrm{FM}}$ (or $j_{\mathrm{HM}}$ ) and $\Delta t$.

For larger damping $\alpha=0.1$ [Fig. 5(b)] the value of $j_{\mathrm{FM}}^{u}=$ $430 \mathrm{GA} / \mathrm{m}^{2}$ (or $\theta_{\mathrm{SH}} j_{\mathrm{HM}}^{u}=17.7 \mathrm{GA} / \mathrm{m}^{2}$ ) exceeds the displayed interval of current densities. Therefore, in Fig. 5(b) all skyrmions traveling along the trajectories which leave the starting valley pass around the antidot I. Then the only way that the skyrmion can reach the valley $(-1,0)$ is by descending from the wall of antidot I after the current pulse is off [light green region in Fig. 5(b). Since the potential of the antidots $V(x, y)$ and the applied electric current have periodicity of the antidot lattice, the points related to the unstable position of the skyrmion are periodically localized throughout the whole antidot lattice. As we have already discussed, the periodicity of skyrmion trajectories can exceed a single lattice constant, which means that a skyrmion can pass to an unstable point several lattice constants away from the starting point. As an example, the discontinuity appearing in the blue region at $(-2,1)$ for $j_{\mathrm{FM}}=240 \mathrm{GA} / \mathrm{m}^{2}$ (or $\theta_{\mathrm{SH}} j_{\mathrm{HM}}=9.9 \mathrm{GA} / \mathrm{m}^{2}$ ) corresponds to the trajectory that passes to the unstable point located between the antidots III and B defined in Fig. 4.

For even larger values of damping, the valley $(-1,0)$ is unreachable within the given range of current densities as is demonstrated in Figs. 5(c) and 5(d) for $\alpha=0.2$ and $\alpha=0.3$, respectively. To summarize, for $\alpha=0.1$, the regions in the parameter plane $\left(\Delta t, j_{\mathrm{FM} / \mathrm{HM}}\right)$ corresponding to the different final positions of the skyrmion are more compact than for the case of small damping $\alpha=0.03$ where the situation is quite chaotic. In contrast, for larger values of damping $\alpha=0.2,0.3$, the region corresponding to the valley $(-1,0)$ is shifted to the large values of current outside of the displayed interval. Therefore, the number of neighboring valleys the skyrmion can be moved to is reduced for $\alpha=0.2,0.3$. Therefore, to maximize the number of reachable valleys by the skyrmion, the value of damping $\alpha=0.1$ is optimal for the given parameters of the antidot lattice and material properties.

For larger values of $j_{\mathrm{FM} / \mathrm{HM}}$ or $\Delta t$, the skyrmion inelastically scatters off the antidots or even annihilates at the antidot edges. The skyrmion annihilation is not captured by the Thiele equation since it is based on the assumption that the magnetization profile of skyrmion is fixed. The transparent gray regions in Fig. 5 highlight the parameters of current pulses which lead to the skyrmion annihilation calculated using micromagnetic simulations. For smaller values of damping, the regions of annihilation cover a significant part of the displayed parameter maps.

\section{System with nonuniform current}

Here we employ a more realistic model of electric current density. Up until now we have assumed that the electric current density is distributed uniformly in the ferromagnetic or heavy metal layer of the heterostructure, i.e., $\mathbf{j}_{\mathrm{FM}}(x, y)=$ $\left(j_{\mathrm{FM}}, 0\right)$ or $\mathbf{j}_{\mathrm{HM}}(x, y)=\left(0, j_{\mathrm{HM}}\right)$. However, since the antidot is a hole in the heterostructure, the current cannot enter the antidot and has to flow around it. Therefore, using the Comsol software package, we have calculated a more realistic nonuniform current distribution taking also an antidot into account.

For the case of STT, we assume a net voltage drop across the ferromagnetic layer along the $x$ direction. The resulting periodic nonuniform current distribution $\mathbf{j}_{\mathrm{FM}}(x, y)$ is shown in Fig. 6(a). Due to the symmetry of the square antidot lattice, the current density averaged along the transversal $(y)$ direction is the same as in the uniform case, i.e., $\frac{1}{a} \int_{0}^{a} \mathbf{j}_{\mathrm{FM}}(x, y) d y=$ $\left(j_{\mathrm{FM}}, 0\right)$ and, as a result of current conservation, it is independent of $x$.

For the case of SOT, we assume a net voltage drop applied in the heavy metal layer along the $y$ direction. The resulting nonuniform current distribution $\mathbf{j}_{\mathrm{HM}}(x, y)$ is shown in Fig. 9(a). Similarly, the current density averaged along the $x$ direction is the same as in the uniform case, i.e., $\frac{1}{a} \int_{0}^{a} \mathbf{j}_{\mathrm{HM}}(x, y) d x=\left(0, j_{\mathrm{HM}}\right)$ and is independent of $y$. 

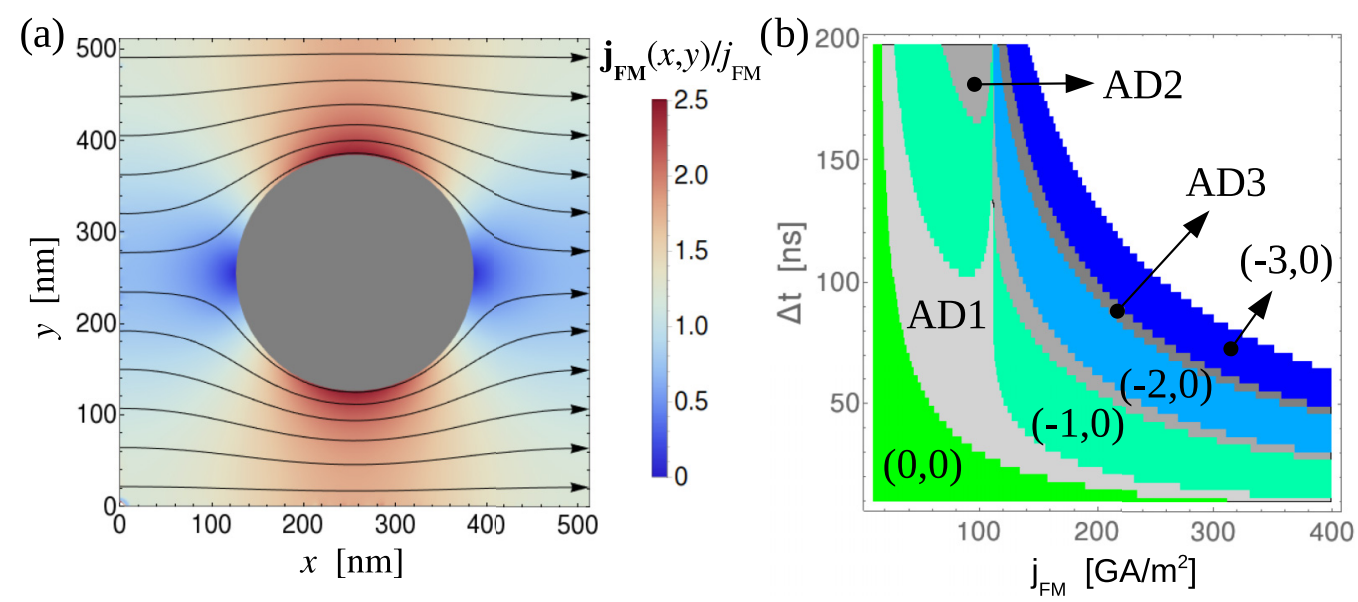

FIG. 6. (a) Distribution of a periodic nonuniform current density $\mathbf{j}_{\mathrm{FM}}(x, y)$ flowing in the ferromagnetic layer depicted in a single unit cell of the antidot lattice. $\mathbf{j}_{\mathrm{FM}}(x, y)$ was calculated using the Comsol package where the antidot hole (gray circle) is assumed to be nonconductive. (b) Map of the final positions of the skyrmion after application of current pulses with nonuniform current density in the undamped system.

We have incorporated this nonuniform current distribution into the micromagnetic simulation and Thiele equation (12) and recalculated the maps of final skyrmion positions in the current pulse parameter plane. In contrast to the previous section where the skyrmion trajectories driven by STT and SOT coincide due to the uniformity of the current distribution, here we show that, assuming a nonuniform current distribution, leads to a different result for each torque mechanism.

\section{Spin transfer torque}

In accordance with the previous subsection, we first discuss the skyrmion dynamics at negligible damping. Combining the expression for the force acting on the skyrmion as a result of STT (7) with the equation of motion (14), we conclude that the electric current $\mathbf{j}_{\mathrm{FM}}$ drives the skyrmion parallel to the current direction. As shown in Fig. 6(a), far away from the antidot, the current flows almost entirely in the $x$ direction and therefore the skyrmion is propelled along this axis just as for the case of the uniform current distribution. However, in the vicinity of the antidot, the current follows its circular edges and supports the motion of the skyrmion around the antidot, which reduces the likelihood that the skyrmion is annihilated.

The map for nonuniform current density and zero damping is shown in Fig. 6(b). This map is very similar to the case of uniform current in Fig. 3(b), but the discontinuity corresponding to the unstable position of skyrmion is shifted to smaller values of current.

The skyrmion transport driven by short current pulses with nonuniform current density is summarized in Fig. 7 for various values of damping. Again, these maps are similar to the maps for uniform current in Fig. 5, but there are some significant differences. In general, as a result of the current flowing around the antidot, the currents needed to move the skyrmion directly through the saddle to the neighboring valley (avoiding the orbiting around an antidot) are smaller. For small damping, $\alpha=0.03$, there are multiple discontinuities corresponding to the unstable positions of skyrmion located between the antidots along the $x$ direction from the starting valley. The light green region corresponding to the valley
$(-1,0)$ is broader, which makes this valley accessible for a larger range of current pulse parameters. Most importantly, the micromagnetic simulations reveal that the annihilation regions are shifted to the larger values of current pulse parameters than for uniform current in Fig. 5. For uniform current, the current flow at one side of the antidot pushes the skyrmion directly towards the antidot edge, while for nonuniform current, as discussed above, the current flowing around the antidot reduces the contact of the skyrmion with the antidot edge. For $\alpha=0.1$ [Fig. 7(b), the annihilation does not affect the regions corre-
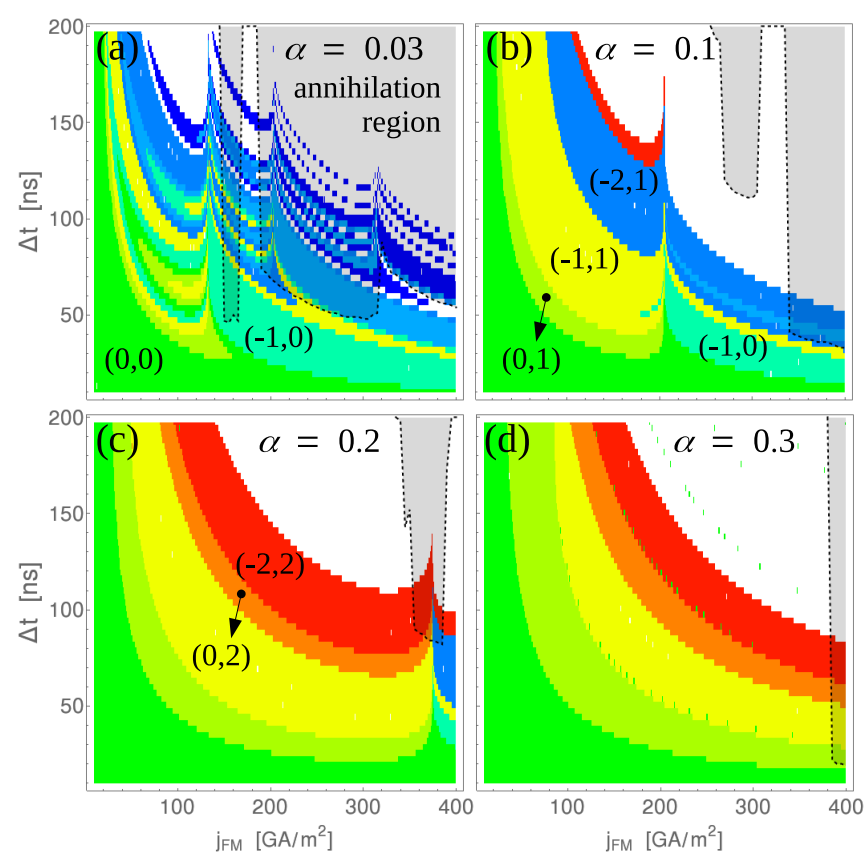

FIG. 7. Final position of the skyrmion driven by STT after the application of nonuniform current pulse with density $j_{\mathrm{FM}}$ and width $\Delta t$. Color regions correspond to the parameters with the same final valley. The coordinate system is shown in Fig. 4. The damping is (a) $\alpha=0.03$, (b) $\alpha=0.1$, (c) $\alpha=0.2$, and (d) $\alpha=0.3$. Shaded region calculated by micromagnetic solver shows the parameters of current pulse at which the skyrmion is annihilated. 


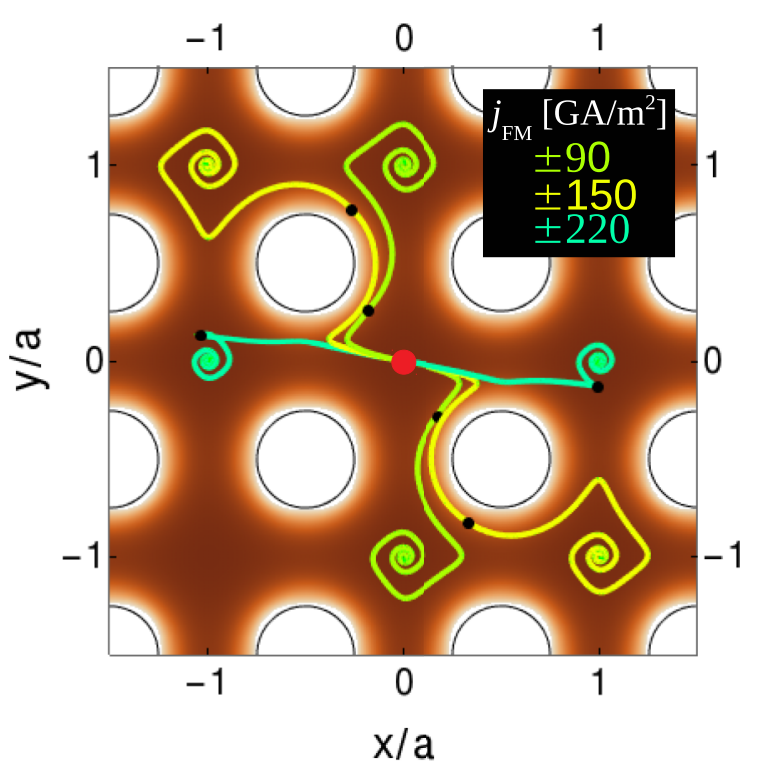

FIG. 8. Skyrmion trajectories of damped system with $\alpha=0.1$, nonuniform current density generating STT, fixed width of current pulse at $\Delta t=50 \mathrm{~ns}$ and varying current densities and polarities. By adjusting a single parameter $\left(j_{\mathrm{FM}}\right)$ of the current pulse, the skyrmion can be transferred to almost all of the neighboring valleys in the longitudinal as well as transversal direction.

sponding to the nearest-neighboring valleys, which makes this map suitable for the well-defined and robust control of the skyrmion motion.

To demonstrate the controlled motion of skyrmions in the antidot lattice we present the response of the skyrmion driven by current pulses applied in the $x$ direction with fixed width $\Delta t=50 \mathrm{~ns}$ and varying density and polarity. The skyrmion trajectories corresponding to $j_{\mathrm{FM}}=$ $\pm 90, \pm 150, \pm 220 \mathrm{GA} / \mathrm{m}^{2}$ are shown in Fig. 8. By tuning a single parameter, current density $j_{\mathrm{FM}}$, the skyrmion starting at the bottom of a valley can be transported to six of the eight neighboring valleys.

\section{Spin orbit torque}

For the case of SOT, the current-generated force (8) within the undamped equation of motion (14) propels the skyrmion orthogonally to the current $\mathbf{j}_{\mathrm{HM}}$ shown in Fig. 9(a). Again, far away from the antidot the skyrmion is propelled by a current parallel to $x$ axis. However, in the vicinity of the antidot, the effect on the skyrmion is very different to the case of the STT. Close to the left half of the antidot, the current causes the skyrmion to be repelled from from the antidot, while close to the right half of the antidot, the current drives the skyrmion towards the antidot. Therefore, we can expect that the attenuation of skyrmion annihilation observed for STT will not be present for the case of SOT.

The map of the final skyrmion positions after applying a current pulse with nonuniform current density and for zero damping are shown in Fig. 9(b). This map is very similar to the case of uniform current in Fig. 3(b), but in contrast to the case of nonuniform current with STT [Fig. 6(b)], the discontinuity corresponding to the unstable position of the skyrmion is shifted to larger values of current. This is caused by the fact that the skyrmion starting at the right side of the antidot is pushed towards the antidot edge by the applied current. In combination with the orbiting motion generated by the antidot potential, the skyrmion is more likely to move around the antidot than to directly pass through the saddles along the $x$ direction. Moreover, the regions in Fig. 9(b) are narrower and shifted to the lower values of pulse widths $\Delta t$ than for the case of uniform current.

As a result of nonzero damping values $\alpha=$ $0.03,0.1,0.2,0.3$, the map in Fig. 9(b) is modified to the maps shown in Fig. 10. Again, there are some significant differences when comparing with the maps for uniform current in Fig. 5. As a result of the skyrmion attraction/repulsion to the antidot caused by the applied current, the currents needed to move the skyrmion directly through the saddle to the neighboring valley (avoiding the orbiting around an antidot) are larger. Then, for $\alpha=0.03$, the discontinuity corresponding to the unstable position of skyrmion is shifted to $\theta_{\mathrm{SH}} j_{\mathrm{HM}} \sim 16 \mathrm{GA} / \mathrm{m}^{2}$.
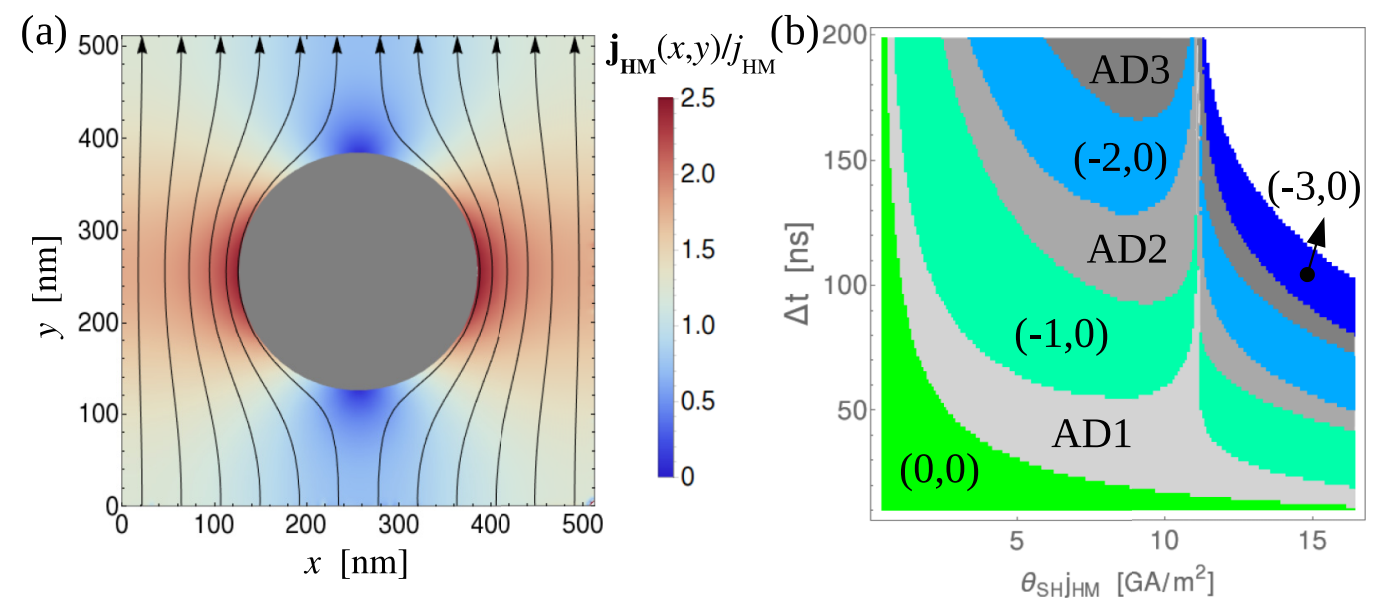

FIG. 9. (a) Distribution of a periodic nonuniform current density $\mathbf{j}_{\mathrm{HM}}(x, y)$ flowing in the heavy metal layer depicted in a single unit cell of the antidot lattice. $\mathbf{j}_{\mathrm{HM}}(x, y)$ was calculated using the Comsol package where the antidot hole (gray circle) is assumed to be nonconductive. (b) Map of the final positions of the skyrmion driven by SOT resulting from current pulses with nonuniform current density in the undamped system. 

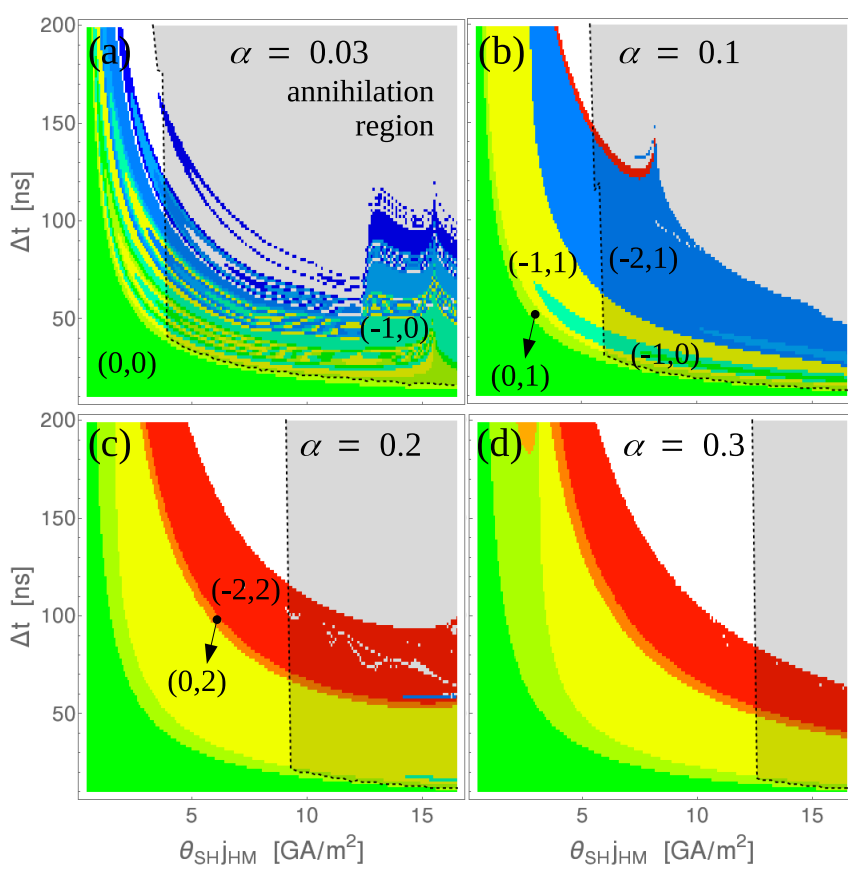

FIG. 10. Final position of the skyrmion driven by SOT after the application of a nonuniform current pulse with density $j_{\mathrm{HM}}$ and width $\Delta t$. Color regions correspond to the parameters with the same final valley. The coordinate system is shown in Fig. 4. The damping is (a) $\alpha=0.03$, (b) $\alpha=0.1$, (c) $\alpha=0.2$, and (d) $\alpha=0.3$. Shaded region calculated by the micromagnetic solver shows the parameters of current pulse at which the skyrmion is annihilated.

The maps for $\alpha=0.1,0.2,0.3$ are very similar to the maps for uniform current in Figs. 5(b)-5(d), i.e., for $\alpha=0.1$, the only way skyrmion can end up in the valley $(-1,0)$ is by passing around the antidot I (e.g., the light blue trajectory in Fig. 11). Unfortunately, as expected, due to the attractive effect of antidots generated by the applied current, the micromagnetically calculated regions of annihilation are a little larger than for the case of uniform current. However, for $\alpha=0.1$ [Fig. 10(b)], the light blue region corresponding to a skyrmion final position located in the valley $(-1,0)$ is extended to the smaller values of current, which makes it possible to achieve the well-defined and robust control of the skyrmion motion driven by SOT.

Similarly to the case of STT (Fig. 8), we demonstrate the controlled motion of skyrmions in the antidot lattice for SOT by showing the skyrmion trajectories generated by current pulses with fixed width $\Delta t=50 \mathrm{~ns}$ and varying density and polarity. The skyrmion trajectories corresponding to $\theta_{\mathrm{SH}} j_{\mathrm{HM}}= \pm 2.9, \pm 3.7, \pm 4.5 \mathrm{GA} / \mathrm{m}^{2}$ are shown in Fig. 11. By tuning a single parameter, current density $j_{\mathrm{HM}}$, the skyrmion starting at the bottom of a valley can be transported to six of the eight neighboring valleys.

\section{SUMMARY}

In this work, we have developed a semianalytical model to determine the skyrmion motion driven by short in-plane current pulses in the presence of a magnetic antidot array. To drive the skyrmion motion we have separately considered

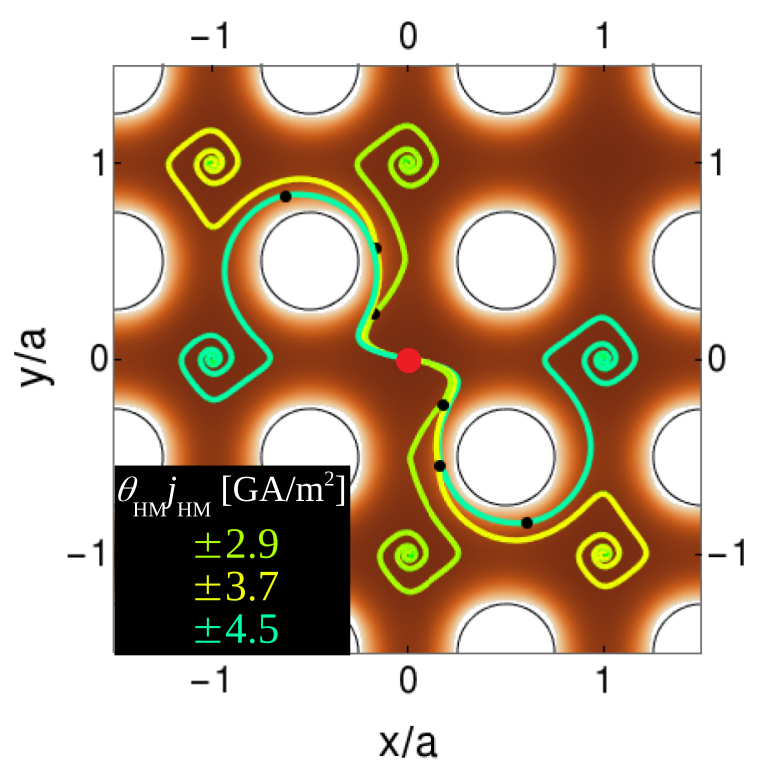

FIG. 11. Skyrmion trajectories of damped system with $\alpha=0.1$, nonuniform current density providing SOT, fixed width of current pulse at $\Delta t=50 \mathrm{~ns}$, and varying current densities and polarities. By adjusting a single parameter $\left(j_{\mathrm{HM}}\right)$ of the current pulse, the skyrmion can be transferred to almost all of the neighboring valleys in the longitudinal as well as transversal direction.

spin transfer torque and spin orbit torque mechanisms. Due to the repulsion of the skyrmion by the antidot edges, the antidot lattice results in an effective potential of attractive valleys located between each four neighboring antidots. For both spin torque mechanisms we demonstrate that skyrmion transport between individual valleys can be controlled by applying a rectangular current pulse with adequate density and width. As a result of the interplay between the antidot potential, skyrmion Hall effect, and nonuniformity of the current, skyrmions can be manipulated in the longitudinal and in the transverse direction with respect to the current flow. We identify two mechanisms determining the final position of the skyrmion: (i) the skyrmion is directly driven by the applied current to the desired valley, (ii) after the current pulse is switched off, the skyrmion relaxes down the antidot wall to the desired valley.

We have calculated maps showing the regions of the current pulse parameters that give a particular final position of the skyrmion after the pulse is switched off. Starting from the bottom of a valley, our calculations show that, by applying an adequate unidirectional current pulse, it is possible to move the skyrmion to almost all of the neighboring valleys horizontally and vertically. Therefore, by using a sequence of electrical current pulses, the magnetic antidot arrays can be used as a medium for well controlled skyrmion motion. Our results are therefore an important step towards skyrmion based devices.

\section{ACKNOWLEDGMENTS}

This study was performed in the frame of the implementation of the project Building-up Centre for advanced materials application of the Slovak Academy of 
Sciences, ITMS Project Code 313021T081 supported by Research \& Innovation Operational Programme funded by the ERDF. Further we acknowledge funding from the Slovak Grant Agency APVV [Grants No. APVV-16-0068(NanoSky) and No. APVV-19-0311(RSWFA)], and Era.Net RUS Plus (TSMFA). J.F. acknowledges the project VEGA 2/0162/18, and J.T. acknowledges the VEGA project $2 / 0150 / 18$.
S.S. acknowledges ETH Zurich Post-Doctoral fellowship and Marie Curie actions for People COFUND program. M.Z. acknowledges funding from the Adam Mickiewicz University Foundation, National Science Centre, Poland Grant No. UMO-2017/27/N/ST3/00419 and No. UMO2018/30/Q/ST3/00416 and National Scholarship Programme of the Slovak Republic.
[1] U. K. Rößler, A. N. Bogdanov, and C. Pfleiderer, Nature (London) 442, 797 (2006).

[2] A. Fert, V. Cros, and J. J. Sampaio, Nat. Nanotechnol. 8, 152 (2013).

[3] S. Mühlbauer, B. Binz, F. Jonietz, C. Pfleiderer, A. Rosch, A. Neubauer, R. Georgii, P. Böni, S. Muhlbauer, B. Binz, F. Jonietz, C. Pfleiderer, A. Rosch, A. Neubauer, R. Georgii, and P. Boni, Science 323, 915 (2009).

[4] O. Boulle, J. Vogel, H. Yang, S. Pizzini, D. de Souza Chaves, A. Locatelli, T. O. Menteş, A. Sala, L. D. Buda-Prejbeanu, O. Klein, M. Belmeguenai, Y. Roussigné, A. Stashkevich, S. Mourad Chérif, L. Aballe, M. Foerster, M. Chshiev, S. Auffret, I. M. Miron, and G. Gaudin, Nat. Nanotechnol. 11, 449 (2016).

[5] I. Dzyaloshinsky, J. Phys. Chem. Solids 4, 241 (1958).

[6] G. Chen, J. Zhu, A. Quesada, J. Li, A. T. N'Diaye, Y. Huo, T. P. Ma, Y. Chen, H. Y. Kwon, C. Won, Z. Q. Qiu, A. K. Schmid, and Y. Z. Wu, Phys. Rev. Lett. 110, 177204 (2013).

[7] S. Woo, K. Litzius, B. Krüger, M.-Y. Im, L. Caretta, K. Richter, M. Mann, A. Krone, R. M. Reeve, M. Weigand, P. Agrawal, I. Lemesh, M. A. Mawass, P. Fischer, M. Kläui, and G. S. D. Beach, Nat. Mater. 15, 501 (2016).

[8] W. Jiang, P. Upadhyaya, W. Zhang, G. Yu, M. B. Jungfleisch, F. Y. Fradin, J. E. Pearson, Y. Tserkovnyak, K. L. Wang, O. Heinonen, S. G. E. te Velthuis, and A. Hoffmann, Science 349, 283 (2015).

[9] W. Koshibae and N. Nagaosa, Sci. Rep. 7, 42645 (2017).

[10] X. Zhang, Y. Zhou, and M. Ezawa, Nat. Commun. 7, 10293 (2016).

[11] J.-V. Kim and M.-W. Yoo, Appl. Phys. Lett. 110, 132404 (2017).

[12] K. Litzius, I. Lemesh, B. Krüger, P. Bassirian, L. Caretta, K. Richter, F. Büttner, K. Sato, O. A. Tretiakov, J. Förster, R. M. Reeve, M. Weigand, I. Bykova, H. Stoll, G. Schütz, G. S. D. Beach, and M. Kläui, Nat. Phys. 13, 170 (2017).

[13] C. Reichhardt, D. Ray, and C. J. Olson Reichhardt, Phys. Rev. B 91, 104426 (2015).

[14] G. Yu, P. Upadhyaya, X. Li, W. Li, S. K. Kim, Y. Fan, K. L. Wong, Y. Tserkovnyak, P. K. Amiri, and K. L. Wang, Nano Lett. 16, 1981 (2016).

[15] J. Sampaio, V. Cros, S. Rohart, A. Thiaville, and A. Fert, Nat. Nanotechnol. 8, 839 (2013).
[16] A. Hrabec, J. Sampaio, M. Belmeguenai, I. Gross, R. Weil, S. M. Chérif, A. Stashkevich, V. Jacques, A. Thiaville, and S. Rohart, Nat. Commun. 8, 15765 (2017).

[17] S. S. P. Parkin, M. Hayashi, and L. Thomas, Science 320, 190 (2008).

[18] P. Milde, D. Köhler, J. Seidel, L. M. Eng, A. Bauer, A. Chacon, J. Kindervater, S. Mühlbauer, C. Pfleiderer, S. Buhrandt, C. Schütte, and A. Rosch, Science 340, 1076 (2013).

[19] G. Finocchio, F. Büttner, R. Tomasello, M. Carpentieri, and M. Kläui, J. Phys. D 49, 423001 (2016).

[20] S. Zhang, A. K. Petford-Long, and C. Phatak, Sci. Rep. 6, 31248 (2016).

[21] D. Suess, C. Vogler, F. Bruckner, P. Heistracher, and F. Slanovc, Sci. Rep. 9, 4827 (2019).

[22] X. Gong, H. Y. Yuan, and X. R. Wang, Phys. Rev. B 101, 064421 (2020).

[23] J. E. Hirsch, Phys. Rev. Lett. 83, 1834 (1999).

[24] L. Liu, C.-F. Pai, Y. Li, H. W. Tseng, D. C. Ralph, and R. A. Buhrman, Science 336, 555 (2012).

[25] S. Saha, M. Zelent, S. Finizio, M. Mruczkiewicz, S. Tacchi, A. K. Suszka, S. Wintz, N. S. Bingham, J. Raabe, M. Krawczyk, and L. J. Heyderman, Phys. Rev. B 100, 144435 (2019).

[26] A. Vansteenkiste, J. Leliaert, M. Dvornik, M. Helsen, F. GarciaSanchez, and B. Van Waeyenberge, AIP advances 4, 107133 (2014).

[27] A. A. Thiele, Phys. Rev. Lett. 30, 230 (1973).

[28] S. Zhang and Z. Li, Phys. Rev. Lett. 93, 127204 (2004).

[29] S. Woo, K. M. Song, X. Zhang, Y. Zhou, M. Ezawa, X. Liu, S. Finizio, J. Raabe, N. J. Lee, S.-I. Kim et al., Nat. Commun. 9, 959 (2018).

[30] I. Makhfudz, B. Krüger, and O. Tchernyshyov, Phys. Rev. Lett. 109, 217201 (2012).

[31] W. Jiang, X. Zhang, G. Yu, W. Zhang, X. Wang, M. B. Jungfleisch, J. E. E. Pearson, X. Cheng, O. Heinonen, K. L. Wang, Y. Zhou, A. Hoffmann, and S. G. E. te Velthuis, Nat. Phys. 13, 162 (2016).

[32] J. Iwasaki, M. Mochizuki, and N. Nagaosa, Nat. Commun. 4, 1463 (2013).

[33] X. S. Wang, H. Y. Yuan, and X. R. Wang, Commun. Phys. 1, 31 (2018).

[34] R. Tomasello, E. Martinez, R. Zivieri, L. Torres, M. Carpentieri, and G. Finocchio, Sci. Rep. 4, 6784 (2014). 